\title{
Visualization of chromosomes and nuclear envelope in living cells for molecular dynamics studies
}

\author{
Takashi Fukada ${ }^{1}$, Kimiko Inoue ${ }^{1}$, Takeshi Urano ${ }^{2}$, and Kenji Sugimoto ${ }^{1}$ \\ ${ }^{1}$ Osaka Prefecture University, Sakai, Osaka and ${ }^{2}$ Nagoya University, Nagoya, Japan
}

BioTechniques 37:552-556(October 2004)

Cell division is a highly dynamic process in which coordinated translocation of various protein molecules is important for precise replication and distribution of intracellular structures such as centrosomes, centromere/ kinetochores, and chromosomes. For example, aurora-B and survivin, known as chromosome passenger proteins, associate with centromere/kinetochores in prometaphase and metaphase, remain in the midzone in anaphase and telophase, and finally migrate to the midbody during the cytokinesis (1). Although immunofluorescent observation of fixed cells shows certain timesections of these protein translocations, it is often difficult to follow the entire behavior of quickly migrating proteins.

Green fluorescent protein (GFP) fusion technique enabled tracking of certain protein in living cells by time-lapse fluorescent microscopy and dramati- cally improved the protein dynamics study. However, some technical inconveniences still associate with the living cell analysis. One of the problems is that it is often difficult to precisely track the progression of mitotic stages unless chromosomes are visualized by fluorescent dye injection or differential interference contrast (DIC) microscopy. It is also difficult to assign the observed GFP signals to certain intracellular structures such as centrosomes and centromere/kinetochores, which are invisible in living unstained cells. Although DIC or phase contrast microscopy can be used to visualize chromosomes (reviewed in Reference 2) in combination with fluorescence microscopy, application of this approach to time-lapse observation usually requires a complex and expensive system that can automatically manage the two different optics. Because of these disadvantages, studies of GFP-fused protein dynamics in living cells usually require supplemental experiments on fixed cells in which some landmark structures are immunologically stained.

To construct a fluorescence-only system for studying protein dynamics in mitosis, we established a human cell line expressing histone $\mathrm{H} 3$ and truncated importin $\alpha$ as fusions to cyan and red fluorescent proteins [enhanced cyan fluorescent protein (ECFP) and DsRed], respectively, to visualize the chromosomes and the nuclear envelope in living cells. These visualized structures served as spatial landmarks for specifying the relative positions of GFP-fused proteins, as well as temporal markers for tracking the progression of cell cycle stages.

Mouse histone H3 cDNA, cloned in pECFP-C1 (BD Biosciences Clontech, Palo Alto, CA, USA), was introduced into a human breast tumor cell line (MDA435). Stably transformed cell lines were isolated by G418 selection, and the expression of ECFP-histone $\mathrm{H} 3$ was confirmed by fluorescent microscopy. A 1-kb cDNA fragment encoding the C-terminal half of human importin $\alpha 1$ (amino acids 251-529) was cloned into pDsRed1-C1 (BD Biosciences Clontech) and transfected into the above transformants with a puromycin-resistance plasmid (pLCpuro), followed by puromycin selection to establish a stable
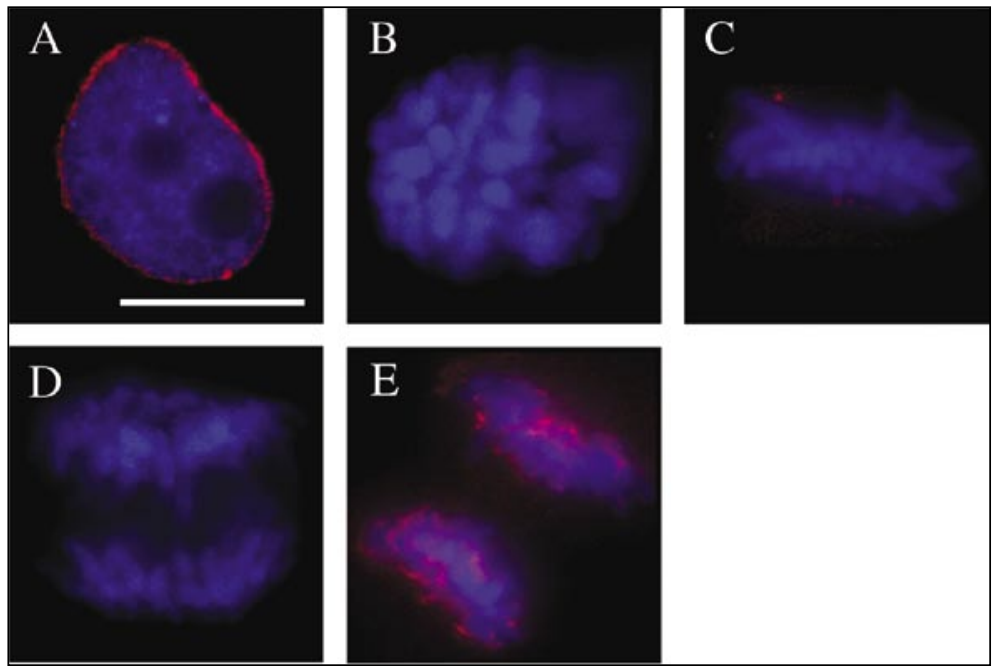

Figure 1. Simultaneous visualization of chromosomes and nuclear envelope in MDA435 cells. MDA435 cells stably expressing enhanced cyan fluorescecnt protein (ECFP)-histone H3 (blue) and DsRed1-importin $\alpha$ (red) were fixed by paraformaldehyde and observed by fluorescent microscopy. (A) Interphase, (B) prophase, (C) metaphase, (D) anaphase, and (E) telophase.

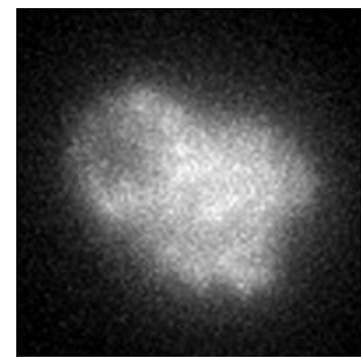

ECFP-histone

(CFP channel)

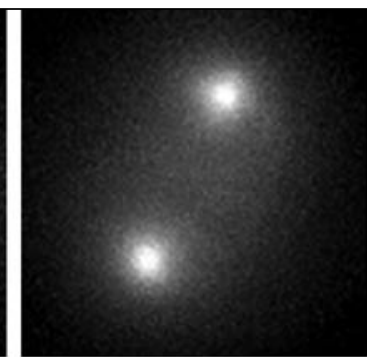

EGFP-aurora-A

(YFP channel)
Figure 2. Specific detection of enhanced green fluorescent (EGFP) fluorescence in MDA-H3/imp cells through yellow fluorescecnt protein (YFP) channel. MDA-H3/imp cells stably expressing EGFP-aurora-A were observed by fluorescent microscopy with an Eclipse TE300 microscope (Nikon, Tokyo, Japan), ORCA-ER digital camera (Hamamatsu Photonics, Hamamatsu, Sizuoka, Japan), Chroma 86006 excitation and emission filters, and Z-axis motor (Ludl Electronic Products, Hawthorne, NY, USA). The filter sets for cyan fluorescecnt protein (CFP) and YFP were used to detect enhanced CFP (ECFP)-histone and EGFP-aurora-A, respectively. 
cell line (MDA-H3/imp) that simultaneously expressed ECFP-histone $\mathrm{H} 3$ and DsRed1-importin $\alpha$. Although the expression of the fluorescent proteins appeared stable, repeated passages of the cells were avoided as long as possible.

MDA-H3/imp cells were fixed by paraformaldehyde and observed by fluorescent microscopy to confirm the proper localization of these fluorescent protein fusions (Figure 1). The nucleus and chromosomes were visualized by ECFP-histone H3 and served as spatial and temporal markers of the structural changes in dividing cells. The localization of DsRed1-importin $\alpha$ indicated the exact time of nuclear envelope breakdown and reformation, offering an additional temporal cue for tracking the cell cycle.

A problem in simultaneous observation of CFP- and GFP-fused proteins was the bleedthrough of CFP fluorescence to GFP channel that might affect the tracking of GFP-fused protein in MDA-H3/ imp cells. We used a filter setting for yellow fluorescent protein (YFP) to detect GFP signals to avoid this problem, at the



B

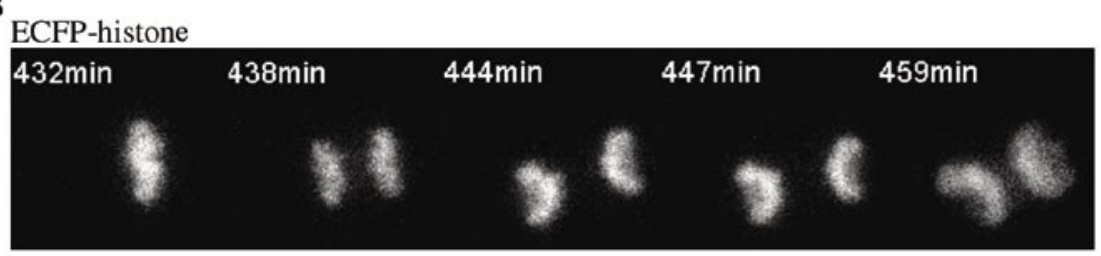

EGFP-survivin (YFP channel)

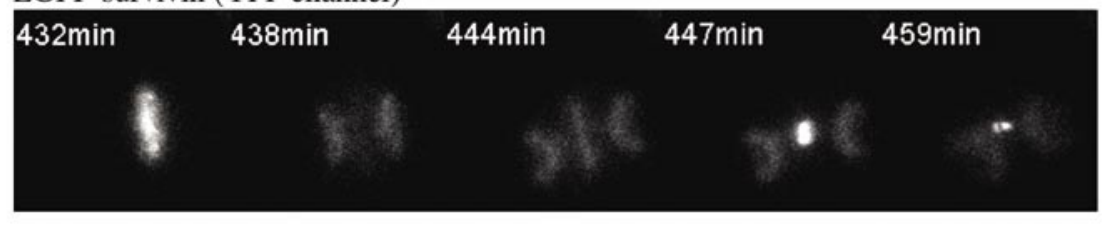

Figure 3. Tracking of green fluorescent protein (GFP)-survivin with visualized chromosomes and nuclear envelope. (A) Migration of enhanced GFP (EGFP)-survivin in living MDA-H3/imp cells in mitosis. Images were captured by LuminaVision software (Mitani, Fukui, Japan) with 3-min intervals through the optics as in Figure 2. Only representative frames are shown (movie of same can be viewed on the BioTechniques web site at http://www.BioTechniques.com/Oct04/FukadaMovie.htm1). Green, EGFP-survivin; blue, enhanced cyan fluorescecnt protein (ECFP)-histone H3; red, DsRed1-importin $\alpha$. (B) Separate images of ECFP-histone and EGFP-survivin between $432 \mathrm{~min}$ and $459 \mathrm{~min}$ as in panel A.

expense of the sensitivity. MDA-H3/imp cells expressing enhanced GFP (EGFP)aurora-A was used to test this approach, since aurora-A was known to localize at cetrosomes in metaphase, well separate from chromosomes. As shown in Figure 2, the fluorescence of ECFP-histone and EGFP-aurora-A was well distinguished by CFP and YFP settings of Chroma 86006 filter sets (Chroma Technology, Rockingham, VT, USA), and the fluorescence of EGFP-aurora-A was enough intense to be readily detected in the YFP channel.

As a model experiment of tracking GFP-fusion protein, we expressed human survivin as a EGFP fusion in MDA-H3/ imp cells and observed its behavior in the living cells. Figure $3 \mathrm{~A}$ showed the timelapse images of the three fluorescent protein fusions simultaneously tracked in a single cell throughout a mitosis (movie of same can be viewed on the BioTechniques web site at http://www.BioTechniques. com/Oct04/FukadaMovie.html). EGFPsurvivin had been excluded from the nucleus during the previous mitosis (data not shown), but reappeared in the nucleus in $\mathrm{G} 2$ /prophase (0 min). It gradually localized to centromere/kinetochores along with the chromosome condensation (219-228 $\mathrm{min}$ ), followed by the nuclear envelope breakdown (231 min). It co-localized with chromosomes during prophase and prometaphase and finally migrated to the midzone in metaphase (429 $\mathrm{min}$ ). In anaphase, it released from the centromere/kinetochores and transiently diffused into the cytosol (435-438 min). It began to accumulate again in the midzone concomitantly with the onset of nuclear envelope reformation in telophase (441 min) and was concentrated into the midbody during the cytokinesis (447-459 min). Only a small amount of EGFP-survivin, if any, was observed in the daughter cell nuclei (444-480 min). These results confirmed the reported changes in survivin localization during a cell division (3).

Previous immunofluorescence analyses of fixed cells (1) did not report the transient release and diffusion of survivin from the centromere/kinetochores in early anaphase. This phenomenon was recently documented by Temme et al. (3) in a time-lapse analysis of living Hela cells expressing DsRed-survivin, although their observation was not tem- 
porally correlated with the processes of chromosome segregation and nuclear envelope reformation. Our live image analysis with the multicolor cell line clearly indicated that survivin diffused into cytosol coincident with the chromosome segregation (435 min) and reappeared in the midzone during the nuclear envelope reformation (441-444 min). The separate images of ECFP-histone and EGFP-survivin shown in Figure 3B suggested that a small amount of survivin remained on chromosomes after their segregation, although we could not completely rule out the possibility that we observed weak bleedthrough of the ECFP fluorescence to the YFP channel.

It is obvious that some kind of spatial and temporal landmarks are necessary for molecular dynamics studies in living cells. However, it is often time-consuming to establish a cell line simultaneously expressing multiple fluorescent protein fusions. In some cases, it may be necessary to tune their expression levels or to change the combination of fluorescent proteins, since DsRed fusions tend to aggregate or abnormally localize, especially when overexpressed. Too little expression of the fusion protein, in contrast, increases the required exposure time to the excitation light and may cause cell death by photopathic damage. This problem is especially important for multicolor time-lapse analysis, since it requires multiple exposures per frame. Our MDA-H3/imp cell line would serve as an excellent premade evaluated hosts for expressing GFP-fused proteins. Further effort to establish other variations of premade hosts for multicolor living cell analysis would contribute to the molecular dynamics studies in mitosis and cell cycle control in the future.

\section{ACKNOWLEDGMENTS}

We thank Dr. Makoto Tachibana for providing mouse histone H3 cDNA. This work was supported by Senri Life Science Foundation.

\section{COMPETING INTERESTS STATEMENT}

The authors declare no conflicts of interest.

\section{REFERENCES}

1.Skoufias, D.A., C. Mollinari, F.B. Lacroix, and R.L. Margolis. 2000. Human survivin is a kinetochore-associated passenger protein. J. Cell Biol. 151:1575-1581.

2. Rieder, C.L. and A. Khodjakov. 2003. Mitosis through the microscope: advances in seeing inside live dividing cells. Science 300:9196.

3.Temme, A., M. Rieger, F. Reber, D. Lindemann, B. Weigle, P. Diestelkoetter-Bachert, G. Ehninger, M. Tatsuka, et al. 2003. Localization, dynamics, and function of survivin revealed by expression of functional survivin-
DsRed fusion proteins in the living cell. Mol. Biol. Cell 14:78-92.

Received 7 April 2004; accepted 22 June 2004.

Address correspondence to Kenji Sugimoto, Graduate School of Agriculture and Biological Sciences, Osaka Prefecture University, 1-1 Gakuen-cho, Sakai, Osaka 599. 8531, Japan.e-mail: sugimoto@biochem. osakafu-u.ac.jp

\title{
High efficiency DNA mutagenesis mediated by using in vitro transcription, DNase I digestion, and RT-PCR
}

\author{
Wen Xin, Da-Wei Huang, and Hui Xiao \\ Chinese Academy of Sciences, Beijing, China
}

BioTechniques 37:556-560 (October 2004)

Site-directed mutagenesis is a powerful tool to generate desired DNA mutants for exploring structure-function relationship. DNA mutants are created by amplifying DNA from a parental DNA template with site-specific mutagenic primers. Several methods have been published to separate the mutants from the parental DNA to achieve higher mutagenesis efficiency, for example digesting methylated parental DNA with $D p n \mathrm{I}$ (References 1-4; http://www.stratagene. com/manuals/200518.pdf), transforming plasmids into specific bacteria strains in which only the mutants can survive (Reference 3; http://www.invitrogen.com/content/sfs/manuals/genetailor_man.pdf), and unique restriction site elimination (5). High mutagenesis efficiency can be achieved with some of those methods. However it is very difficult to achieve $100 \%$ mutagenesis efficiency, because all of these methods are based upon discrimination.

Here we describe a highly efficient mutagenesis strategy. This strategy has a few unique features. PCR primer containing $\mathrm{T} 7$ promoter sequence and a unique type IIS restriction enzyme site upstream of the mutagenesis sequence is used to generate template for in vi- tro transcription. After that, all DNAs are completely digested with the most effective DNase (DNase I) without discrimination. RNA is used as template to generate the desired mutants. With this method, we consistently achieve $100 \%$ mutagenesis efficiency with single or double point mutations.

The whole scheme of this mutagenesis method was described in Figure 1. It was a seven-step procedure. Step 1: the preparation of DNA template for in vitro transcription (Figure 1a). PCR mutagenic forward primers contained the $\mathrm{T} 7$ promoter sequence, the Bsm BI recognition site, and the target-specific sequence with single or double point mutations, and the PCR reverse primer contained only the BsmBI recognition site and the target-specific sequence. Fifty microliters of PCR contained 2.5 U of Pyrobest $^{\mathrm{TM}}$ DNA polymerase (Takara, Toyko, Japan), $5 \mu \mathrm{L}$ of $10 \times$ Pyrobest PCR buffer (Takara), $400 \mu \mathrm{M}$ of dNTPs, $20 \mathrm{ng}$ of DNA template $[5.6 \mathrm{~kb} \mathrm{pCMV} / \mathrm{cyto} / \mathrm{myc} /$ green fluorescent protein (GFP); Invitrogen, Carlsbad, CA, USA), and $100 \mathrm{ng}$ of each PCR primer. PCR procedure was as follows: $94^{\circ} \mathrm{C}$ for $5 \mathrm{~min}$ for one cycle for predenaturation, 30 cycles of denaturation at $94^{\circ} \mathrm{C}$ for $30 \mathrm{~s}$, annealing and ex- 